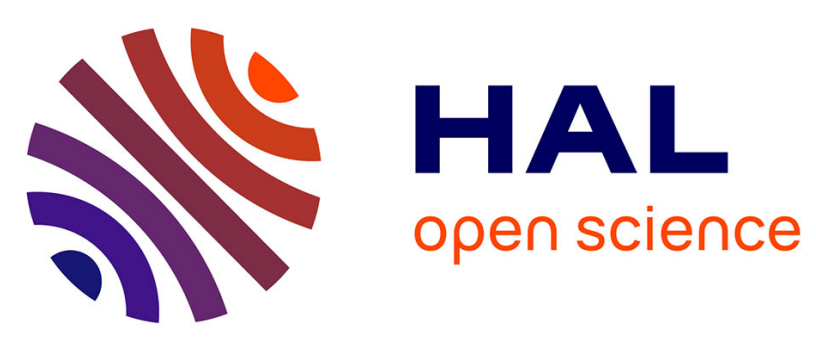

\title{
Translation Control of a Fleet Circular Formation of AUVs under Finite Communication Range
}

\author{
Lara Briñon Arranz, Alexandre Seuret, Carlos Canudas de Wit
}

\section{To cite this version:}

Lara Briñon Arranz, Alexandre Seuret, Carlos Canudas de Wit. Translation Control of a Fleet Circular Formation of AUVs under Finite Communication Range. CDC 2009 - 48th IEEE Conference on Decision and Control, Dec 2009, Shanghai, China. pp.6. hal-00403253

\section{HAL Id: hal-00403253 https://hal.science/hal-00403253}

Submitted on 9 Jul 2009

HAL is a multi-disciplinary open access archive for the deposit and dissemination of scientific research documents, whether they are published or not. The documents may come from teaching and research institutions in France or abroad, or from public or private research centers.
L'archive ouverte pluridisciplinaire HAL, est destinée au dépôt et à la diffusion de documents scientifiques de niveau recherche, publiés ou non, émanant des établissements d'enseignement et de recherche français ou étrangers, des laboratoires publics ou privés. 


\title{
Translation Control of a Fleet Circular Formation of AUVs under Finite Communication Range
}

\author{
Lara Briñón Arranz* \\ lara.brinon-arranz@inrialpes.fr \\ Alexandre Seuret** \\ alexandre.seuret@gipsa-lab.inpg.fr \\ Carlos Canudas de Wit** \\ carlos.canudas-de-wit@gipsa-lab.inpg.fr
}

\author{
INRIA Rhône-Alpes* \\ NeCS Team, Grenoble, France
}

\author{
Department of Automatic Control, GIPSA-Lab** \\ NeCS Team, Grenoble, France
}

\begin{abstract}
This work proposes a control algorithm to stabilize a circular formation of AUVs tracking a time-varying center. We also consider the problem of uniform distribution of all the agents along the circle from two approaches: all-to-all and limited communication. We tackle with this communication constraint using a cooperative control which includes the Laplacian matrix of the communication graph (fixed or distance-dependent). The system was implemented in computer simulation, accessible though $\mathrm{Web}^{1}$.
\end{abstract}

\section{INTRODUCTION}

This paper treats the problem of formation translation in multi-agent control under limited communication range. In particular, we propose an extension of the control proposed in [1], [2] to the case where the center of the cycle formation is time-varying. This is studied under different set-ups: allto-all, fixed, and range-dependent communication graphs.

This problem is pertinent to some applications where the agents should perform collaborative tasks requiring the formation to displace towards an a priori unknown direction. For instance, in source seeking applications, the formation is displaced in the source gradient direction (which is computed on-line, and instrumented as an additional outer loop). Translations of the formation can be seen here as a first step toward more general formation motions including rotation and contraction/expansion.

Formation control has been extensively studied in [1], [2], [3], [4], [5], [6], [7] among many others. These studies concern circular and parallel formations [1], [2], [6], [7], but also motions of formation induced by flocking [8], [9]. One strategy to produce formation motions (i.e. flight formations) is the virtual-leader approach [3], where an agent is designed as being the leader, and then a suitable interdistance (and orientation) is set between agents. The motion of the formation results from the motions of the leader. Although it is possible to create circular formations via a particular pursuit graph as suggested in the work of [4], it turn out however, impossible to apply this ideas to the problem of circular formation if the formation is desired to be keep "rigid" while moving. In the context of the source

\footnotetext{
${ }^{1}$ Simulations are accessibles in the CONNECT project web at http://www.lag.ensieg.inpg.fr/connect/
}

seeking problem of underwater vehicles advocated here, it is necessary to keep AUVs (uniformly distributed) formation during the source search to avoid unnecessary energy waist, and to produce efficient search motions. To the knowledge of the authors, the problem of circular formation control with a time-varying (almost arbitrarily) center, while keeping the formation rigid has not bee addressed so far.

Another difficulty in the underwater fleet formation problem is due to the transmission of information in a marine media. Communication between agents is confronted with several difficulties such as signal distorsion and interference, doppler effect, etc. Communication in shallow water amplifies these limitations in particular. In this work, we assume that the communication between each agent is "good enough" within a particular range specific to the application. Therefore, the previously described moving circular formation control will be studied under such a limited-range communication assumptions, where the communication graph depends the agent location [8],[10],[11].

In the present work we first show that tracking a moving circle is not possible with constant linear velocities. We thus relax this assumption by using one additional control, and we show that after a suitable change of coordinates, this problem can be solved by a new feedback law yielding global asymptotic stability. We show that the stability conditions not only hold for all-to-all communication but also for fixed limited communication graph yielding uniformly distributed formation. We also devise a control law for the case of rangedependent graph, and provides some simulation showing the asymptotic convergence.

\section{A. Problem formulation}

Consider a set of $N$ agents (vehicles), in which each agent $k=1, \ldots, N$ has the following constrained dynamics:

$$
\begin{aligned}
\dot{\mathbf{r}}_{k} & =v_{k} e^{i \theta_{k}} \\
\dot{\theta}_{k} & =u_{k}
\end{aligned}
$$

where $\mathbf{r}_{k}$ is the position vector, $\theta_{k}$ the heading angle and $v_{k}, u_{k}$ are the control inputs, as illustrated in Figure 1. This is the standard agent model commonly used the literature to model AUVs restricted kinematics [4], [12], [13] [1], [7], [6], 


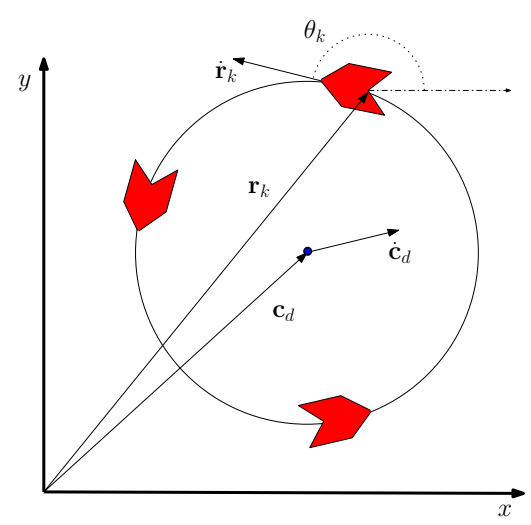

Fig. 1. Illustration of the problem formulation.

[14], [2] and [15]. It corresponds to a kinematic unicycle fitting with model properties subject to a simple nonholonomic constraint, adequate for the underwater vehicles.

The problem is to design a control law such that the group of AUVs forms a circle that tracks the time-varying center motion $\mathbf{c}_{d}(t)$ as described in Figure 1. $\mathbf{c}_{d}(t)$ is considered here as an external reference. The circle radius $R$ and the rotation velocity $\omega_{0}$ are given parameters. Moreover, an additional objective is to achieve a uniform distribution of all the agents along the circle (i.e., the difference between headings of adjacent vehicles is $2 \pi / N$ ), under two different cases:

1) Fixed communication graph

2) Limited range time-varying communication graph

Notation. A complex number $\mathbf{z}$ is written in boldface and is expressed as $\mathbf{z}=x_{z}+i y_{z}$ where $i^{2}=-1$ and where $x_{z}=\operatorname{Re}\{z\}$ and $y_{z}=\operatorname{Im}\{z\}$ correspond to the real and the imaginary part of $\mathbf{z}$. For compactness in the notation, we use the following operator $\left\langle\mathbf{z}_{1}, \mathbf{z}_{2}\right\rangle=\operatorname{Re}\left\{\overline{\mathbf{z}}_{1}^{T} \mathbf{z}_{2}\right\}$ where $\overline{\mathbf{z}}_{1}^{T}$ represents the conjugate transpose of $\mathbf{z}_{1}$. Note that the real part (respectively the imaginary part) of a complex number $\mathbf{z}$ can be written as $\langle\mathbf{z}, 1\rangle$ (and respectively $\langle\mathbf{z}, i\rangle$ ). Thus, for any complex numbers $\mathbf{z}_{1}$ and $\mathbf{z}_{2}$, the equality $\left\langle\mathbf{z}_{1}, i\right\rangle\left\langle\mathbf{z}_{2}, 1\right\rangle-\left\langle\mathbf{z}_{1}, 1\right\rangle\left\langle\mathbf{z}_{2}, i\right\rangle=\left\langle\mathbf{z}_{1}, i \mathbf{z}_{2}\right\rangle$ holds. Also, the notation $|\mathbf{z}|=\langle\mathbf{z}, \mathbf{z}\rangle^{1 / 2}$ and $\angle \mathbf{z}$ denotes the magnitude and the argument of the complex number $\mathbf{z}$.

\section{Translation Control for a Moving Circle}

\section{A. Preliminaries}

Some previous works in the field of coordinated control and specifically of planar collective motions, use the kinematic model in which each vehicle moves in the plane subject to planar steering control, which is our model (1) with constant velocity $v=1$. In [1], the authors suggest a control law for stabilization to a circular formation center at a particular and constant $\mathbf{c}_{d}^{0}$. It corresponds to the center of mass and is obtained by solving a consensus algorithm. The control law uses the relative position vector from the center to vehicle $k$ defined as $\tilde{\mathbf{r}}_{k}=\mathbf{r}_{k}-\mathbf{c}_{d}^{0}=\frac{1}{N} \sum_{j=1}^{N}\left(\mathbf{r}_{k}-\mathbf{r}_{j}\right)$. For such a formation, the authors propose the following theorem:
Theorem 1 (Leonard et al. [1]) Consider the vehicle model (1) with $v_{k}=1, \forall k$. Then the control law:

$$
u_{k}=\omega_{0}\left(1+\kappa\left\langle\tilde{\boldsymbol{r}}_{k}, \dot{\boldsymbol{r}}_{k}\right\rangle\right)
$$

where $\kappa>0$ is a scalar gain, ensures that all the agents converge to a circular formation centered at $c_{d}^{0}$ and of radius $\rho_{0}=\left|\omega_{0}\right|^{-1}$.

Proof: The proof is based the Lyapunov function:

$$
S(\mathbf{r}, \theta)=\frac{1}{2} \sum_{k=1}^{N}\left|v e^{i \theta_{k}}-i \omega_{0} \tilde{\mathbf{r}}_{k}\right|^{2}
$$

The details can also be found in [6].

Remark 1 Note that when $S(\boldsymbol{r}, \theta)=0$, the dynamics of the agents satisfy the differential equation $\dot{\boldsymbol{r}}_{k}-i \omega_{0} \tilde{\boldsymbol{r}}_{k}=0$. As the center $\boldsymbol{c}_{d}^{0}$ is fixed, $\dot{\boldsymbol{r}}_{k}=\dot{\tilde{\boldsymbol{r}}}_{k}$ and the previous equation means that $\dot{\tilde{\boldsymbol{r}}}_{k}-i \omega_{0} \tilde{\boldsymbol{r}}_{k}=0$ which corresponds to a circular motion around $\boldsymbol{c}_{d}^{0}$ with an angular velocity $\omega_{0}$.

\section{B. Fundamental limitations}

These previous results are only applicable to the case of a fixed formation center, $\mathbf{c}_{d}$. In this situation, it is sufficient to design a control law such that the velocity of all the agents is constant (i.e. $v_{k}=1, \forall k$ ). However, when it comes to the case of a time-varying center $\mathbf{c}_{d}(t)$, the mechanical equations for the combined motion of a rotation and a translation of the rigid body leads to a contradiction with the choice of constant velocity of the agents (see for instance the example of the wheel motion).

Hence a new strategy which tackles the objectives needs to be developed. The velocity $v_{k}$ becomes a new and necessary control input to overcome this mechanical constraint. Then, in the sequel, the variables $\left(v_{k}, u_{k}\right)$ and $\left(\mathbf{r}_{k}, \theta_{k}\right)$, respectively, are the inputs and the state of the agents. In the latter, the notations $\mathbf{r}$ and $\theta$ denote the vectors containing the position and headings of all the agents.

\section{Translation Control Design}

We assume that the first and second time-derivative of $\mathbf{c}_{d}$ are known and bounded. Inspired the Theorem 1, to keep the circular formation, all the agents must satisfy the equation $\dot{\tilde{\mathbf{r}}}_{k}-i \omega_{0} \tilde{\mathbf{r}}_{k}=0$, where $\tilde{\mathbf{r}}_{k}=\mathbf{r}_{k}-\mathbf{c}_{d}$. However since the center is moving, the velocities $\mathbf{r}_{k}$ and $\tilde{\mathbf{r}}_{k}$ are not the same anymore. The previous Lyapunov function will thus not be useful for reaching the time-varying formation.

To simplify the problem, we introduce the change of variables, shown in Figure 2, to express the relative velocity in a more convenient representation to obtain a circular formation. The new variable $\psi_{k}$ and the constant $v_{0}>0$ are defined such that:

$$
\dot{\tilde{\mathbf{r}}}_{k}=\dot{\mathbf{r}}_{k}-\dot{\mathbf{c}}_{d}=v_{0} e^{i \psi_{k}}
$$

and $\psi_{k}$ and $v_{0}$ are given as:

$$
\begin{aligned}
& \psi_{k}=\arctan \frac{\left\langle\dot{\mathbf{r}}_{k}-\dot{\mathbf{c}}_{d}, i\right\rangle}{\left\langle\dot{\mathbf{r}}_{k}-\dot{\mathbf{c}}_{d}, 1\right\rangle}+\epsilon \pi \\
& v_{0}>\sup _{t \geq 0}\left\{\left|\dot{\mathbf{c}}_{d}(t)\right|\right\}
\end{aligned}
$$




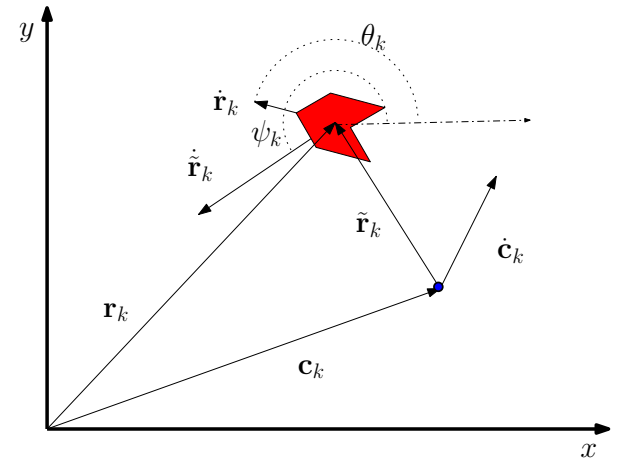

Fig. 2. Vehicles Model

where $\epsilon=0$ if $\left\langle\dot{\mathbf{r}}_{k}-\dot{\mathbf{c}}_{d}, 1\right\rangle>0$ and 1 otherwise. The control input to stabilize the relative position to the circular motion is $\dot{\psi}_{k}$. This new system of coordinates allows us to consider a circular formation centered at 0 . We are now in the situation of a fixed center which corresponds to the previous problem solved in Theorem 1. A new control law is proposed in the following theorem:

Theorem 2 Consider a function $\boldsymbol{c}_{d}: \mathbb{R} \rightarrow \mathbb{C}$ twice differentiable, with bounded first and second time-derivatives and the radius of desired formation $R>0$. Let the control parameters be such that $v_{0}>\sup _{t \geq 0}\left\{\left|\dot{\boldsymbol{c}}_{d}(t)\right|\right\},\left|\omega_{0}\right|=v_{0} / R$, and $\kappa>0$. Then the control law:

$$
\begin{aligned}
v_{k} & =\left|v_{0} e^{i \psi_{k}}-\dot{\boldsymbol{c}}_{d}\right| \\
u_{k} & =\left(1-\frac{\left\langle\dot{\boldsymbol{r}}_{k}, \dot{\boldsymbol{c}}_{d}\right\rangle}{\left\langle\dot{\boldsymbol{r}}_{k}, \dot{\boldsymbol{r}}_{k}\right\rangle}\right) \dot{\psi}_{k}-\frac{\left\langle\dot{\boldsymbol{r}}_{k}, i \ddot{\boldsymbol{c}}_{d}\right\rangle}{\left\langle\dot{\boldsymbol{r}}_{k}, \dot{\boldsymbol{r}}_{k}\right\rangle}
\end{aligned}
$$

with

$$
\left\{\begin{array}{l}
\dot{\psi}_{k}=\omega_{0}\left(1+\kappa\left\langle\boldsymbol{r}_{k}-\boldsymbol{c}_{d}, v_{0} e^{i \psi_{k}}\right\rangle\right) \\
\psi_{k}(0)=\arctan \frac{\left\langle\dot{\boldsymbol{i}}_{k}(0)-\dot{\boldsymbol{c}}_{d}(0), i\right\rangle}{\left\langle\dot{\boldsymbol{r}}_{k}(0)-\dot{\boldsymbol{c}}_{d}(0), 1\right\rangle}+\epsilon_{k} \pi
\end{array}\right.
$$

where $\epsilon_{k}=0$ if $\left\langle\dot{\boldsymbol{r}}_{k}(0)-\dot{\boldsymbol{c}}_{d}(0), 1\right\rangle>0$ and 1 otherwise, makes all the agents defined by (1) converge to a circular motion of radius $R$, and of center the time-varying reference $\boldsymbol{c}_{d}$. The direction of rotation is determined by the sign of $\omega_{0}$.

Proof: The convergence to the formation is analyzed with the Lyapunov function:

$$
S(\tilde{\mathbf{r}}, \psi)=\frac{1}{2} \sum_{k=1}^{N}\left|\dot{\mathbf{r}}_{k}-i \omega_{0} \tilde{\mathbf{r}}_{k}\right|^{2}
$$

The differentiation of $S(\tilde{\mathbf{r}}, \psi)$ leads to:

$$
\dot{S}(\tilde{\mathbf{r}}, \psi)=\sum_{k=1}^{N}\left\langle\text { omega }_{0} \tilde{\mathbf{r}}_{k}, v_{0} e^{i \psi_{k}}\right\rangle\left(\omega_{0}-\dot{\psi}_{k}\right)
$$

Introducing the control requirement (6), the derivative of the Lyapunov function thus satisfies $\dot{S}(\tilde{\mathbf{r}}, \psi) \leq 0$. Therefore $S(\tilde{\mathbf{r}}, \psi)$ is an acceptable Lyapunov function for this system and the agents asymptotically reach the circular formation centered at $\mathbf{c}_{d}$ and of radius $R=v_{0} /\left|\omega_{0}\right|$.
The next step of the proof concerns the design of the control inputs of the original system. Considering (1a), it is easy to see that $v_{k}$ and $\theta_{k}$ are given by:

$$
v_{k}=\left|\dot{\mathbf{r}}_{k}\right| \text { and } \tan \theta_{k}=\frac{\left\langle\dot{\mathbf{r}}_{k}, i\right\rangle}{\left\langle\dot{\mathbf{r}}_{k}, 1\right\rangle}
$$

The control input $v_{k}$ is thus straightforwardly given by (5a). A more particular attention is addressed to the input $u_{k}$. To fit with the model (1), we derive the expression of $\tan (\theta)_{k}$. The properties of the operator $\langle\cdot, \cdot\rangle$ allows obtaining the relation $\dot{\theta_{k}}=\left\langle\ddot{\mathbf{r}}_{k}, i \dot{\mathbf{r}}_{k}\right\rangle /\left\langle\dot{\mathbf{r}}_{k}, \dot{\mathbf{r}}_{k}\right\rangle$. Noting that $\ddot{\mathbf{r}}_{k}=\ddot{\tilde{\mathbf{r}}}_{k}+\ddot{\mathbf{c}}_{d}=i \dot{\tilde{\mathbf{r}}} \dot{\psi}_{k}+$ $\ddot{\mathbf{c}}_{d}=i\left(\dot{\mathbf{r}}_{k}-\dot{\mathbf{c}}_{d}\right) \dot{\psi}_{k}+\ddot{\mathbf{c}}_{d}$ the control law (5b) is obtained.

We have designed a control law for the agents to follow a time-varying circular formation. Note that this control law has singular points when $v_{k}=\left|\dot{\mathbf{r}}_{k}\right|$ is zero. To understand this singularity, consider the exemple of the cycloid whose first derivative is not defined at some instants. This constraint fits with the choice of underwater vehicles. This singular point occurs if there exists a time $t_{c}$ such that:

$$
\angle \dot{\mathbf{c}}_{d}\left(t_{c}\right)=\psi_{k}\left(t_{c}\right)+\pi \quad \text { and } \quad\left|\dot{\mathbf{c}}_{d}\left(t_{c}\right)\right|=v_{0}
$$

However condition (4) of Theorem 2 ensures that this situation is avoided since $\left|\dot{\mathbf{c}}_{d}\left(t_{c}\right)\right| \neq v_{0} \quad \forall t_{c}>0$.

Remark 2 Consider the vehicle model (1) with the circle center fixed, the the angles $\psi_{k}$ and $\theta_{k}$ are equal, and the control law (5) is the same control as in Theorem 1:

$$
\begin{gathered}
v_{k}=v_{0} \text { and } u_{k}=\dot{\psi}_{k}=\omega_{0}\left(1+\kappa\left\langle\tilde{\boldsymbol{r}}_{k}, v_{0} e^{i \psi_{k}}\right\rangle\right) \\
\text { III. COOPERATIVE CONTROL DESIGN UNDER } \\
\text { COMMUNICATION CONSTRAINS }
\end{gathered}
$$

\section{Cooperative Control Design Under COMMUNiCATION CONSTRAINS}

This section is dedicated to the problem of homogenizing the distribution of the agents along the circle. The desired control law is decentralized, i.e. the use of a global controller who organizes the distribution of the agents around the circle is not permitted. As a first step of research, this section tackle the problem of limited communication range. Considering limited communication means that each agent may receive information from only some of the other agents [10]. It is well known that designing collaborative controllers leads to more difficulties than in the case of all-to-all communications.

\section{A. Preliminaries on Graph Theory}

This paragraph presents some basic tools of graph theory. When an agent $k$ communicates with an agent $j$ both agents are called neighbors. The set of neighbors of agent $k$ is denoted by $\mathcal{N}_{k}$. The communication topology for the groups of agents can be represented by means of a graph $G(V, E)$ where $V=\{1,2, \ldots, N\}$ is the set of vertices (agents) and $E=\left\{(k, j): j \in \mathcal{N}_{k}\right\}$ the set of edges (communication links) such that $(k, j) \in E$ if agent $k$ communicates with agent $j$. The adjacency matrix $\mathbf{A}=\left[a_{k j}\right]$ is the $N \times N$ matrix given by $a_{k j}=1$ if $(k, j) \in E$ and $a_{k j}=0$ otherwise. The degree $d_{k}$ of vertex $k$ is defined as the number of its neighboring vertices. Let $\Delta$ be the $N \times N$ diagonal matrix of $d_{k}$ 's. The Laplacian of $G$ is the matrix $L=\Delta-A$. For 


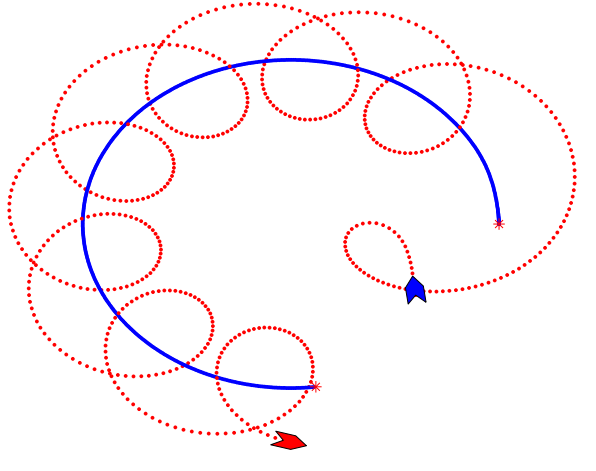

Fig. 3. Simulation of an agent with the control law (5). The trajectories of $\mathbf{c}_{d}$ and of the agent are respectively the continuous and the dashed lines.

an undirected graph, ( $j$ is a neighbor of $k$ if and only if $k$ is a neighbor of $j$ ), the Laplacian matrix is symmetric positive semidefinite [6].

\section{B. Fixed communication graph}

This paragraph is an application of the result of [1] to the case of a time-varying formation center. A potential function is added to the formation control law to achieve the uniform distribution. In the case of a fixed communication structure, we assume $G$ is undirected because we consider the communication is bidirectional between two AUVs. Then a constant Laplacian matrix $\mathbf{L}$ describes the communications links between agents. In this way, we propose the following Theorem:

Theorem 3 Consider a function $\boldsymbol{c}_{d}: \mathbb{R} \rightarrow \mathbb{C}$ twice differentiable, with bounded first and second time-derivatives and the radius of desired formation $R>0$. Let the control parameters be such that $v_{0}>\sup _{t \geq 0}\left\{\left|\dot{\boldsymbol{c}}_{d}(t)\right|\right\},\left|\omega_{0}\right|=v_{0} / R$, and $\kappa>0$. Let $G$ be circulant and $\boldsymbol{L}$ be the corresponding Laplacian matrix. Then the control law (5) now with:

$$
\left\{\begin{array}{l}
\dot{\psi}_{k}=\omega_{0}\left(1+\kappa\left\langle\boldsymbol{r}_{k}-\boldsymbol{c}_{d}, v_{0} e^{i \psi_{k}}\right\rangle\right)-\frac{\partial U}{\partial \psi_{k}} \\
\psi_{k}(0)=\arctan \frac{\left\langle\boldsymbol{r}_{k}(0)-\dot{\boldsymbol{c}}_{d}(0), i\right\rangle}{\left\langle\dot{\boldsymbol{r}}_{k}(0)-\dot{\boldsymbol{c}}_{d}(0), 1\right\rangle}+\epsilon \pi
\end{array}\right.
$$

where $\epsilon=0$ if $\left\langle\dot{\boldsymbol{r}}_{k}(0)-\dot{\boldsymbol{c}}_{d}(0), 1\right\rangle>0$ and 1 otherwise, and:

$$
U(\psi)=-\frac{K}{N} \sum_{m=1}^{[N / 2]} \frac{1}{2 m^{2}}\left\langle e^{i m \psi}, \boldsymbol{L} e^{i m \psi}\right\rangle
$$

makes all the agents defined by (1) converge to a circular motion of radius $R=v_{0} /\left|\omega_{0}\right|$ and of center the time-varying reference $c_{d}$. Moreover the curve-phase arrangement is a critical point of $U(\psi)$. For $K>0$, the set of curve-phase arrangements that are synchronized modulo $2 \pi / N$ is locally exponentially stable.

Proof: The proof uses the La Salle Invariance principle applied to the function $\left\langle e^{i m \psi}, \mathbf{L} e^{i m \psi}\right\rangle / 2 m^{2}$ which is zero for $\psi$ synchronized modulo $2 \pi / m$ and positive otherwise. In this way, is combined the previous circular law with a gradient control term which leads to (7). Consider the new Lyapunov function:

$$
V(\tilde{\mathbf{r}}, \psi)=\kappa S(\tilde{\mathbf{r}}, \psi)+U(\psi)
$$

The differentiation of the Lyapunov function along the trajectories of the agents leads to:

$$
\dot{V}(\tilde{\mathbf{r}}, \psi)=\sum_{k=1}^{N}\left[\kappa<\omega_{0} \tilde{\mathbf{r}}_{k}, \dot{\tilde{\psi}}_{k}>\left(\omega_{0}-\dot{\psi}_{k}\right)+\frac{\partial U}{\partial \psi_{k}} \dot{\psi}_{k}\right]
$$

In [1], the requirement that the potential function $U(\psi)$ is chosen such that $\langle\nabla U, \mathbf{1}\rangle=0$ is introduced. This means that the potential function is invariant with respect to any rotation centered at $\mathbf{c}_{d}$ [1]. Noting that this is equivalent to $\omega_{0} \sum_{k=1}^{N} \frac{\partial U}{\partial \psi_{k}}=0$, the derivative of $V$ is now expressed as follows:

$$
\dot{V}(\tilde{\mathbf{r}}, \psi)=\sum_{k=1}^{N}\left[\kappa<\omega_{0} \tilde{\mathbf{r}}_{k}, \dot{\tilde{\mathbf{r}}}_{k}>-\frac{\partial U}{\partial \psi_{k}}\right]\left(\omega_{0}-\dot{\psi}_{k}\right)
$$

Applying the control law (7), it is easy to see that $\dot{V}(\tilde{\mathbf{r}}, \psi) \leq 0$. The control law (7) ensures that a minimum of $U$ which corresponds to a particular distribution is reached. However we have no guarantee of reaching the global minimum but only a local one.

Remark 3 Theorem 3 does not exclude convergence to formations which corresponds to other critical points of $U(\psi)$ [14].

Remark 4 If the graph $G$ is complete, then the set of curvephase arrangements that are balanced modulo $2 \pi / m$ is a global maximum of $\left\langle e^{i m \psi}, \boldsymbol{L} e^{i m \psi}\right\rangle / 2 m^{2}$ in the reduced space of relative curve-phases; Therefore the set of curvephase arrangements that are balanced modulo $2 \pi / N$ is a global maximum of $U(\psi)$ in the reduced space of relative curve-phases; this is asymptotically stable for $K>0$ [14]. Moreover if $K<0$ the control law of Theorem 3 forces convergence to the synchronized circular formation [6].

\section{Range-dependent communication graph}

In the previous subsection, a control law ensures that the agents reach a circular formation centered at the time varying position $\mathbf{c}_{d}$. It also distributes the agents in a particular way. However as shown in [14], there is no guarantee that the formation is uniform along the circle in the case of fixed communication graphs. Moreover in practice, considering fixed communication graphs is not realistic since the two linked agents could be very far away from one another. As in the case of underwater communication, the quality of the link is strongly affected by the distance between two agents [3], [16], [17], [18], it might be more interesting to consider distance-dependent communication graph.

Hence, a communication area is introduced. Assume this area for any agent is defined by $\rho$ which is the critical communication distance given by the characteristics of the communication devices and of the environment of the AUVs. 
It is assumed to be the same for all AUVs. The condition to get a communication between $k$ and $j$ is expressed as:

$$
k \in \mathcal{N}_{j} \text { and } j \in \mathcal{N}_{k} \Longleftrightarrow\left|\mathbf{r}_{k}-\mathbf{r}_{j}\right|^{2} \leq(2 \rho)^{2}
$$

Based on the definitions presented in section III-A, a timevarying Laplacian matrix $\mathbf{L}(t)$ is defined as:

$$
L_{k, j}= \begin{cases}d_{k}, & \text { if } k=j \\ -1, & \text { if }\left|\mathbf{r}_{k}-\mathbf{r}_{j}\right|^{2} \leq(2 \rho)^{2} \\ 0 & \text { otherwise }\end{cases}
$$

where $d_{k}$ is the degree of vertex $k$. In such a situation, the following theorem holds.

Theorem 4 Consider a function $\boldsymbol{c}_{d}: \mathbb{R} \rightarrow \mathbb{C}$ twice differentiable, with bounded first and second time-derivatives and the radius of desired formation $R>0$. Let the control parameters be such that $v_{0}>\sup _{t \geq 0}\left\{\left|\dot{\boldsymbol{c}}_{d}(t)\right|\right\},\left|\omega_{0}\right|=v_{0} / R$, $\kappa>0$ and:

$$
v_{0} /\left|\omega_{0}\right|<\rho
$$

Then the control law (5) with (7) and (8) ensures that all agents reach the circular formation centered at $\boldsymbol{c}_{d}(t)$ of radius $R$. Moreover the uniform distribution of the agents along the circle is achieved.

Proof: Considering that all the agents asymptotically reach the circle centered at $\mathbf{c}_{d}$ and of radius $v_{0} /\left|\omega_{0}\right|$. Consider $\epsilon>0$ such that $v_{0} /\left|\omega_{0}\right|+\epsilon<\rho$. There exits a time $t_{L}$ such that the distance between all agents is less than $v_{0} /\left|\omega_{0}\right|+\epsilon$ and consequently less than $\rho$. The communication graph is thus complete. By vertue of the potential function $U(\psi)$, the formation is uniformly distributed along the circle.

Remark 5 Note that condition (10) is restrictive since the uniform distribution can also obtained for smaller radius $v_{0} /\left|\omega_{0}\right|$. However this Theorem constitutes a first result on the case of circular formation around a time-varying center with range dependent communication graph.

Besides these limitations described above, Theorem 4 allows obtaining a unform distribution whatever the critical distance $\rho$ by managing with the ratio of the relative velocity of each agent $v_{0}$ and angular velocity of the formation $\omega_{0}$.

Remark 6 From the geometric constraints the minimal distance between two agents $k$ and $j$ lying in the circle is given by $d=R \sin \frac{\psi_{k}-\psi_{j}}{2}$. In the case of uniform distribution, the minimal value of $\psi_{k}-\psi_{j}$ is given by $2 \pi / N$ and so a necessary condition for the agents to communicate in such situation is $\rho>R \sin \frac{\pi}{N}$. If not, the formation is not uniform all over the circle but only on a section of the circle as it will be shown in the simulations.

\section{Simulation Results}

This section presents the simulation of the AUVs whose dynamics are defined in (1). The time-varying center of the formation describes a circle around the origine. The vector $\mathbf{c}_{d}$ is taken as $\mathbf{c}_{d}^{0} e^{\omega_{1} t}$ where $\mathbf{c}_{d}^{0}=3$ and $\omega_{1}=27.7 e^{-3}$ and $\left|\dot{\mathbf{c}}_{d}\right|=83.3 e^{-3}$ satisfying the assumption of Theorems 2, 3

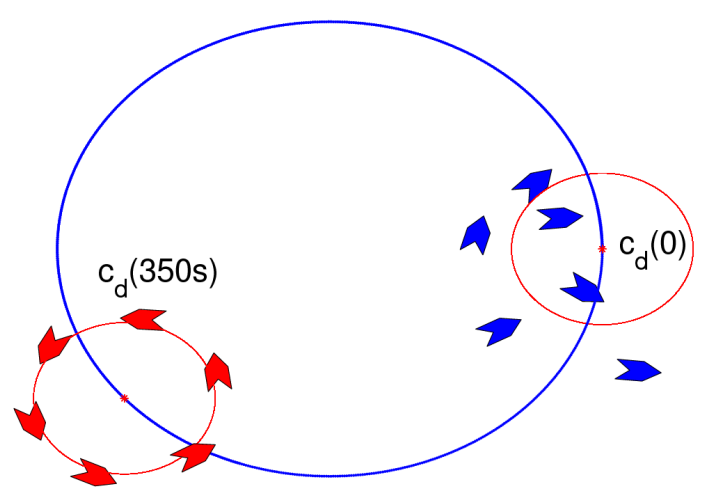

(a)

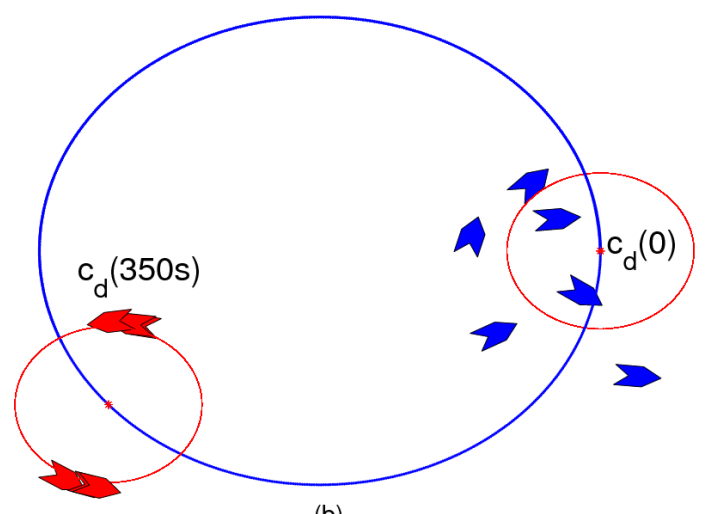

(b)

Fig. 4. Simulation of six agents with the controller of Theorem 3 in the case of two fixed communication graph : (a) all-to-all (b) ring communication. Each figure shows two snapshots. The blue one represents the initial condition and the red one represents the stable final state.

and 4. In the simulation the controller parameters are $\kappa=1$, $v_{0}=1$ and $\omega_{0}=1$. The control parameter to achieve the uniform distribution is $K=0.1$.

Figure 3 shows the trajectory of only one agent governed by the control law defined in Theorem 2 . The snapshot shows trajectories of one vehicle's trajectory during the motion. The tracking of the circle is achieved for all random initial conditions (position and heading of agents).

Figure 4 shows the trajectories of all agents tracking the time-varying formation centered at $\mathbf{c}_{d}$ in the cases of a complete communication graph (a) and of a ring communication graph (b). One can see that in (a) the formation is uniformly distributed along the circle of radius $R=v_{0} /\left|\omega_{0}\right|$. This is not the case in (b). The agents converge to a formation which corresponds to a local minimum of the potential function but not to the global one.

Figure 5 shows the trajectories of agents under rangedependent communication. The three simulations start from the same random initial conditions of positions and headings of all the agents. It is represented in blue $\left(\mathbf{c}_{d}(0)\right)$. After $30 \mathrm{~s}$ all simulations show that all the agents converge to the circular formation. At this time, in the first one, the geometric constraint $R \sin (\pi / N)<\rho$ is not satisfied then the agents do not achieve the uniform distribution all over the circle but only on a section of the circle. For the 


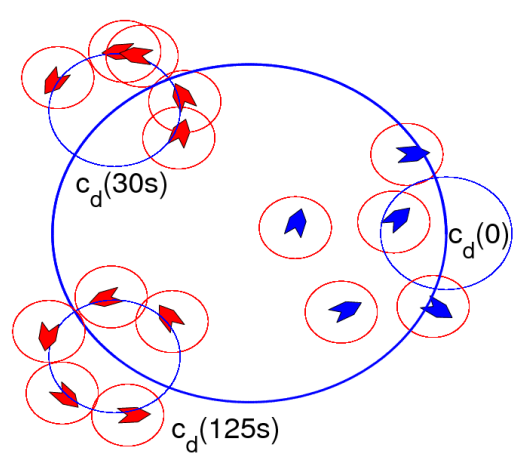

(a) $\rho=0.55<\frac{v_{0}}{\left|\omega_{0}\right|} \sin (\pi / N)$

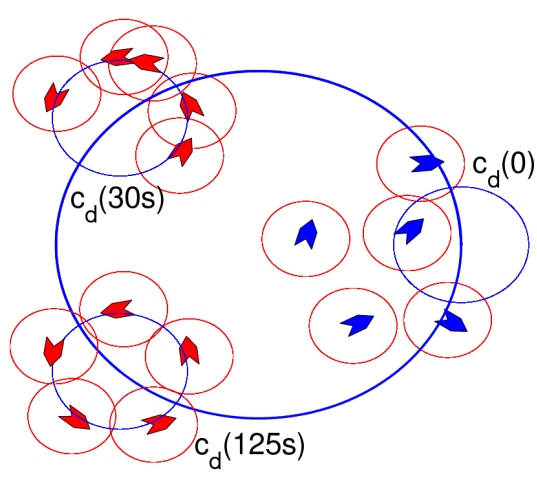

(b) $\rho=0.65>\frac{v_{0}}{\left|\omega_{0}\right|} \sin (\pi / N)$

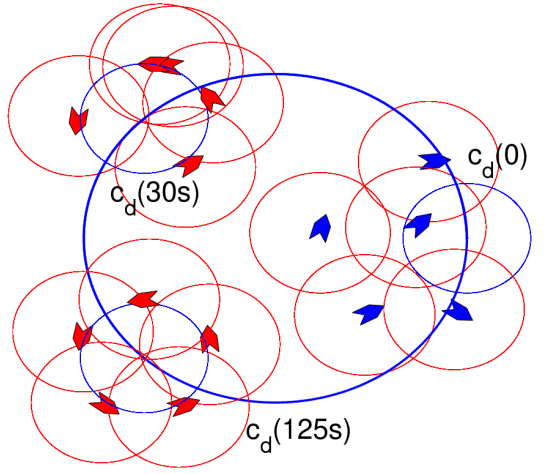

(c) $\rho=1.1>v_{0} /\left|\omega_{0}\right|$

Fig. 5. Simulation of five agents with the controller designed in Theorem 4 and range-dependent communication. Each figure shows three snapshots. The blue one represents the initial condition and the reds one represent a intermediate state and the stable final state.

second one, the previous geometric constraint is satisfied then the agents achieve the uniform distribution along the circle. In the last one, there exists all-to-all communication since condition (10) is satisfied. Thanks to the time-varying communication graph is complete from a certain instant, the uniform distribution is also achieved $\left(\mathbf{c}_{d}(125 s)\right)$.

\section{Conclusions}

We presented a translation control law that stabilizes a circular formation tracking a time-varying center of the circle. This center is a given reference which is known for all the agents. Moreover this paper proposes a cooperative control algorithm to achieve the uniform distribution of the agents along the moving circular formation. This algorithm integrates with the translation control a potential function which reachs its minimum in the desired uniform configuration. This potential function is designed taking into account the communication constraints between agents. The result of this combination is a cooperative control of a planar particle model with limited communication to track a time-varying reference.

At this time, it is assumed that all agents have perfect knowledge of the position of the center $\mathbf{c}_{d}$ and its first and second derivatives. One can consider this assumption as a very restrictive one. However this constitues a first step of our research and further developments would consider a cooperative algorithm which will avoid this assumption. To include collaborative algorithms in the control design would be a step towards source tracking.

\section{ACKNOWLEDGEMENTS}

This research is supported by FeedNetBack project and CONNECT project. FeedNetBack is a recently accepted EU STREP project FP7: ICT-2007.3.7 and the CONNECT project is funded by ANR (Agence Nationale de la Recherche), PSIRob program PSIROB06-174215

\section{REFERENCES}

[1] N. E. Leonard, D. A. Paley, F. Lekien, R. Sepulchre, D. M. Frantatoni, and R. E. Davis, "Collective motion, sensor networks and ocean sampling," Proceedings of the IEEE, vol. 95, no. 1, pp. 48-74, January 2007.
[2] R. Sepulchre, D. A. Paley, and N. E. Leonard, "Stabilization of planar collective motion with limited communication," IEEE Transactions on Automatic Control, vol. 53, pp. 706-719, 2008.

[3] R. L. Raffard, C. J. Tomlin, and S. P. Boyd, "Distributed optimization for cooperative agents: Application to formation flight," 43rd IEEE Conference on Decision and Control, 2004.

[4] J. A. Marshall, M. E. Broucke, and B. A. Francis, "Formations of vehicles in cyclic pursuit," IEEE Transactions on Automatic Control, vol. 49, no. 11, pp. 1963-1974, 2004.

[5] P. Ögren, M. Egerstedt, and X. Hu, "A control lyapunov function approach to multiagent coordination," IEEE Transactions on Automatic Control, vol. 18, no. 5, October 2002.

[6] R. Sepulchre, D. A. Paley, and N. E. Leonard, "Stabilization of planar collective motion: All-to-all communication," IEEE Transactions on Automatic Control, vol. 52, pp. 811-824, 2007.

[7] R. Sepulchre, D. Paley, and N. E. Leonard, "Graph laplacian and lyapunov design of collective planar motions," Proceedings of International Symposium on Nonlinear Theory and its Applications, 2005.

[8] R. Olfati-Saber and R. M. Murray, "Consensus problems in networks of agents whit switching topology and time-delays," IEEE Transactions on Automatic Control, vol. 49, no. 9, pp. 1520-1533, September 2004.

[9] C. W. Reynolds, "Flocks, herds, and schools: A distributed behavioral model," in Computer Graphics, vol. 21, no. 4, July 1987.

[10] R. Olfati-Saber, J. A. Fax, and R. M. Murray, "Consensus and cooperation in networked multi-agent systems," Proceedings of the IEEE, vol. 95, no. 1, pp. 215-233, January 2007.

[11] W. Ren and R. W. Beard, "Consensus seeking in multi-agents systems under dynamically changing interaction topologies," IEEE Transactions on Automatic Control, vol. 50, pp. 655-661, 2005.

[12] J. Yu, S. M. L. Valle, and D. Liberzon, "Rendezvous without coordinates," Proceedings of the 47th IEEE Conference on Decision and Control, December 2008.

[13] J. Cochran and M. Krstic, "Source seeking with a nonholonomic unicycle without position measurements and with tuning of angular velocity part i: Stability analysis," in Proceedings of the 46th IEEE Conference on Decision and Control, 2007.

[14] D. A. Paley, N. E. Leonard, and R. Sepulchre, "Stabilization of symmetric formations to motion around convex loops," Systems \& Control Letters, vol. 57, pp. 209-215, 2008.

[15] R. Sepulchre, D. A. Paley, and N. E. Leonard, "Group coordination and cooperative control of steered particles in the plane," Lecture Notes in control and Information Sciences, 2006.

[16] L. Brekhovskikh and Y. P. Lysanov, Fundamentals of Ocean Acoustics. Springer, 2003.

[17] A. Quazi and W. Konrad, "Underwater acoustic communicaitions," IEEE Comm. Magazine, 1982.

[18] M. Stojanovic, "Recent advances in high rate underwater acoustic communications," IEEE J. Oceanic Eng., 1996. 